> Objective: the aim of the study was to evaluate the efficacy of continuous positive airway pressure (CPAP) for the treatment of obstructive sleep apnea in the elderly.

Method: a comprehensive search for qualified clinical trials was performed on April, 2016. Basic demographic information of enrolled subjects, study design, survival rate, cardiovascular events, quality of life scores, and neurocognitive data were extracted for analysis.

Results: A total of seven clinical trials were included in this meta-analysis, in which untreated elderly patients exhibited worse survival rate than those with CPAP $(0 \mathrm{R}=2.22,95 \% \mathrm{Cl}=1.64$ to 3.01 , $P<0.00001)$. Treated elderly patients exhibited less cardiovascular risk than those without CPAP （RR=0.49, 95\% Cl=0.36 to $0.66, P<0.00001)$ and $a$ statistically significant improvement on all the domains of Quebec Sleepiness Questionnaire, supported by pooled weighted mean difference. Furthermore, CPAP treatment partially improved the cognitive functions.

Conclusion: CPAP treatment achieves improvements in decreasing mortality and controlling cardiovascular events and exhibits few effects on neurocognitive function. Further large-scale, well-designed interventional investigation is needed. <

Key words: Elderly, OSA, CPAP, CVD, Cognition, Meta-analysis.

\section{Introduction}

Obstructive sleep apnea (OSA) is a chronic disease characterized by common sleep-related respiratory disorder, affecting up to $20 \%$ of the general population $[1,2]$. The clinical manifestations of sleep apnea are not often obvious, but OSA is highly prevalent among the elderly; it affects $30 \%$ to $80 \%$ of people over 65 years old

\title{
Effects of continuous positive airway pressure on elderly patients with obstructive sleep apnea: a meta- analysis
}

Binhong Yan ${ }^{1 *}$, Yongmei Jin ${ }^{2 * *}, Y_{i} \mathrm{Hu}^{1}$, Shu Li ${ }^{1}$

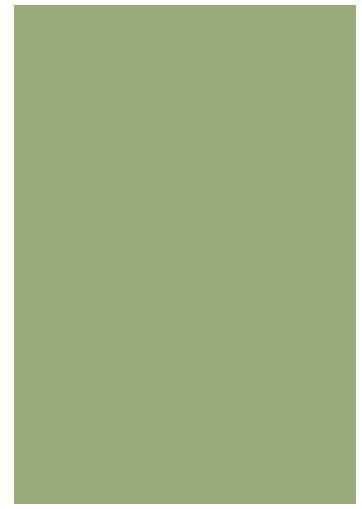

$[1,3]$. The long-term OSA can result in severe daytime sleepiness, reduced social functioning and quality of life, and increased risk of traffic accidents

[4-6], whereas the short-term sequelae are associated with traffic and workplace accidents [7, 8]. Accumulating studies document that OSA can lead to oxidative stress [9], systemic inflammation [10], endothelial dysfunction [11], high blood pressure [12], increased risk of cardiovascular disease [13, 14], stroke [15], and neurocognitive dysfunction [16]. In the elderly (age > 60 years), the prevalence of OSA syndrome is increased and the symptoms can be conflated with the functional impairments of ageing [17]. Takama and Kurabayashi [18] reported that the presence of OSA is a strong predictor of fatal cardiovascular events in elderly patients with cardiovascular disease (CVD). Martinez-Garcia et al. [19] reported that untreated, severe OSA in elderly patients is associated with an approximately two-fold increase in cardiovascular mortality compared with elderly patients without OSA. Continuous positive airway pressure (CPAP) is established as the firstline standard therapy for OSA, which is the most cost-effective treatment for severe or symptomatic forms of OSA [20]. Recent research has shown beneficial effects from CPAP treatment, such as resolving disordered breathing during sleep, normalizing oxygen saturation, and improving nocturnal and daytime symptoms. Moreover, CPAP treatment has demonstrated a positive effect on blood pressure levels [21], and 
significantly decreased cardiovascular complications, including the incidence of hypertension, coronary artery disease, and chronic heart failure, arrhythmia, stroke, and aortic dissection in patients with moderate to severe OSA and CVD [5, 22-24]. CPAP improves cognitive difficulties and reverses brain changes.

Recently, several prospectively and retrospectively randomized clinical trials were performed to evaluate the efficacy of CPAP in elderly with OSA compared with those not treated with CPAP. Some studies estimated the overall survival and cardiovascular risk, whereas others validated the neurocognitive function. In this context, a meta-analysis is urgently needed to confirm whether CPAP can treat OSA among the elderly.

\section{Methods}

\section{Searching design}

The following Mesh terms and keywords on "obstructive sleep apnea," "continuous positive airway pressure," and "elderly" were systematically searched through the database PubMed on April 10, 2016. We restricted the published language to English and the published year from 1 January 2000 to the present. In addition, the reference lists of previously published review articles were manually searched.

\section{Inclusion criteria}

Eligible references were selected carefully based on the following criteria: (1) the age of enrolled patients was $>65$ years old; (2) patients with historically or newly diagnosed moderate to severe OSA; (3) studies comparing the clinical outcomes between the CPAP treated and untreated groups; (4) information collected including survival rate, CVD risk rate, cognitive function, sleepiness score, and quality of life, along with the $95 \%$ confidential indexes $(\mathrm{Cls})$.

\section{Data extraction and bias assessment}

Two reviewers independently extracted all the data with the use of standardized data-abstraction forms. Disagreements were resolved by consensus. The following information was collected from each study, although some information was not contained in some trials: trial name, first author, year of publication, number of patients enrolled in each trial, patient characteristics (including median age, gender, smoking history, OSA severity, and disease history), study design (blinded or not), and outcomes. Outcomes included survival rate, CVD risk rate, and their $95 \% \mathrm{Cls}$, as well as cognitive function (such as episodic, short-term memory, and executive function) and quality of life (Quebec Sleep Questionnaire).

The risk of bias of the included studies was assessed from six domains including sequence generation, allocation concealment, blinding, incomplete outcome data, selective outcome reporting, and other issues.

\section{Statistical analysis}

All the analysis was performed in RevMan 5.3 software. The pooled odds ratios (ORs) for survival rate and pooled risk ratios (RRs) for CVD risk rate were calculated. The weighted mean difference was used

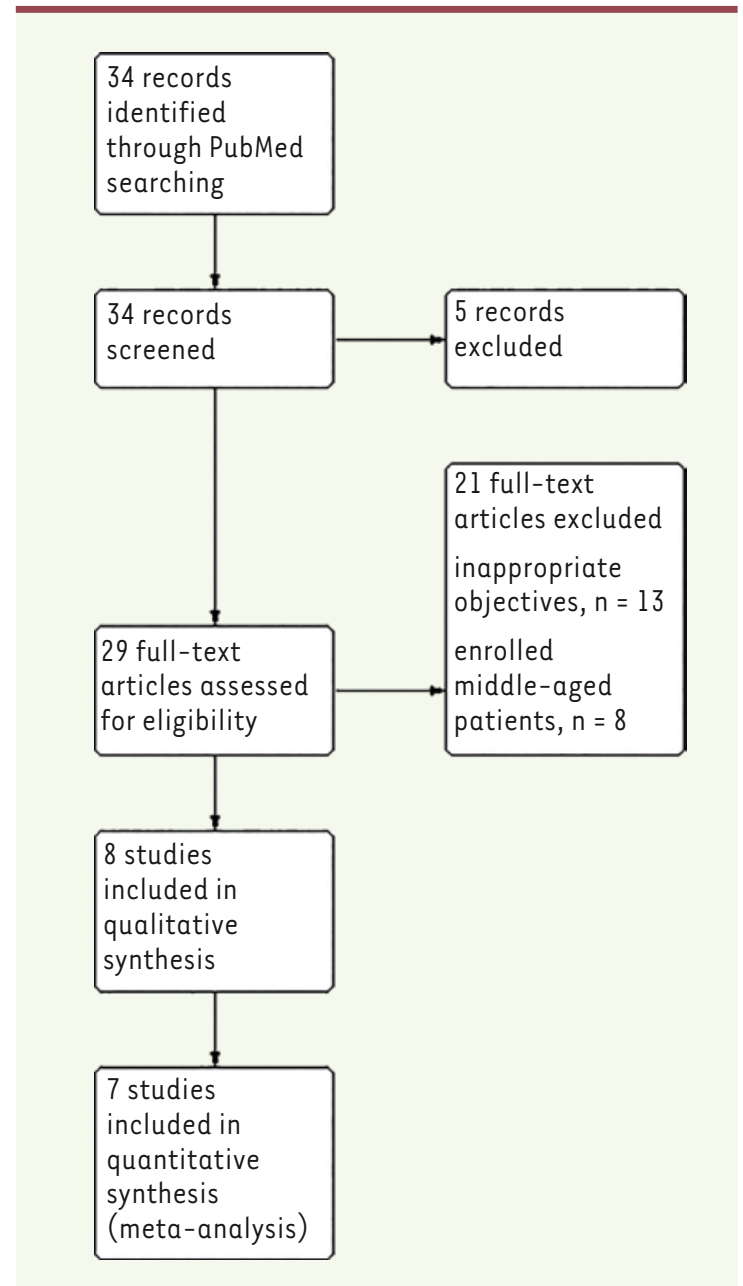

Figure 1. Searching diagram and inclusion flowchart.

to evaluate the effect of CPAP on the quality of life and neurocognitive test. The statistical heterogeneity between trials was evaluated by the chi-squared $\mathrm{Q}$-test based on the fixed-effect model. When the $P$ value of the $Q$-test was less than 0.1 , or when $I^{2}$ was greater than $50 \%$, this result represented heterogeneity between clinical trials. Then, a random effect model was used to accommodate the heterogeneity.

\section{Results}

\section{Searching results and Studies enrollment}

After systematic database searched and manual review of reference lists in the eligible studies, a total of 34 publications were preliminarily included. On the basis of the selection criteria, seven clinical trials comparing the clinical outcomes, cognitive function, and quality of life between treated and untreated CPAP were included in the present meta-analysis $[19,25-30]$. The flow of this search strategy is shown in Figure 1. 


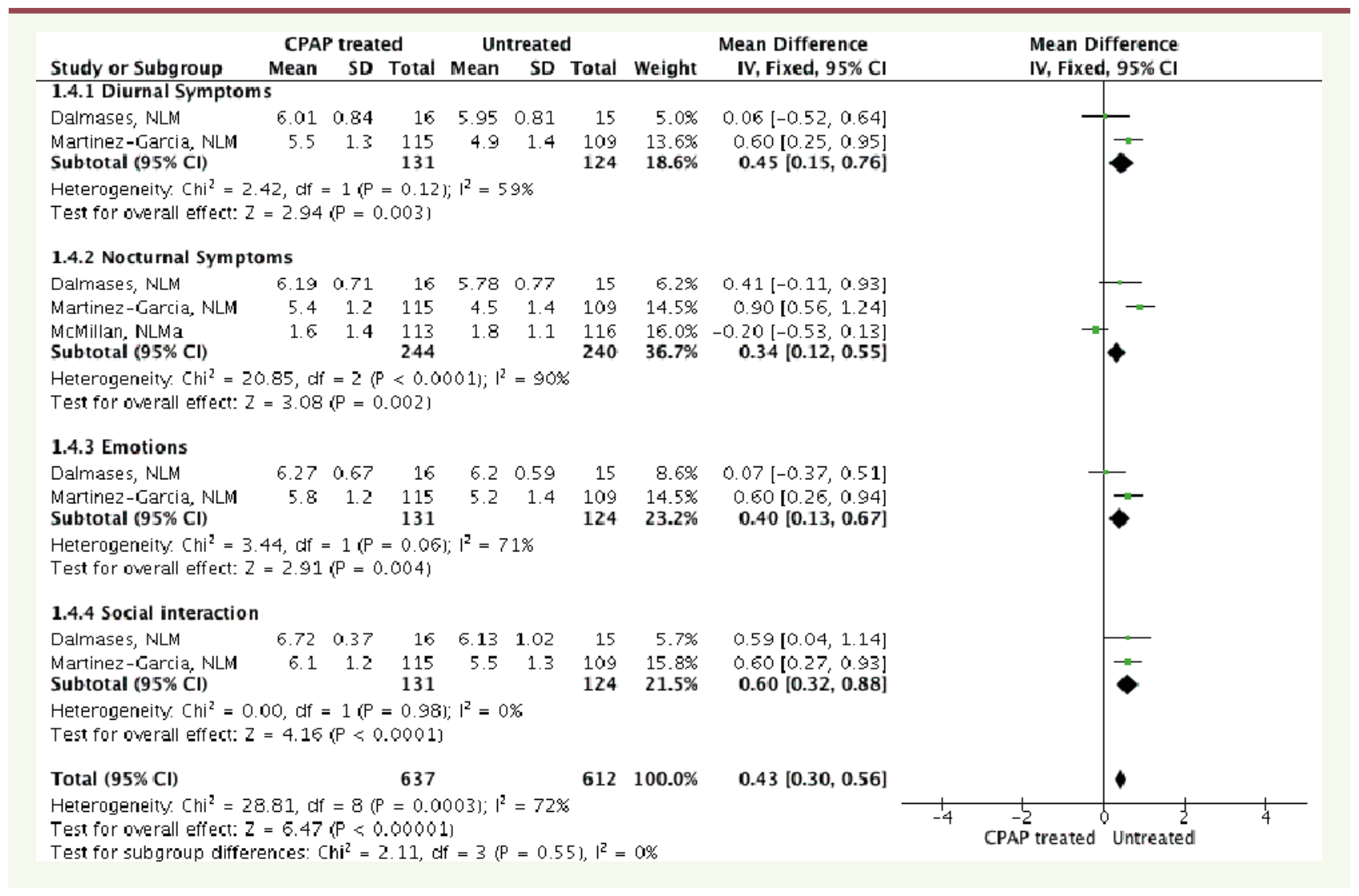

Supplementary Figure 1. Forest plot for Quality of Life.

The characteristics of all these seven clinical trials, such as the basic information of the enrolled patients, their performance status, OSA status, and disease history, are listed in Table 1.

\section{Risk of bias Evaluation}

The bias evaluation is exhibited in Figure 2, in which three studies performed well in the randomization and allocation processes [27-29] and three other studies did not mention the details [19, 25, 26]. Only one study exhibited high concern in the selection bias [30]. Most studies showed unclear risk in blinding for lacking of specific clarification and only McMillan et al. reported the blinding process both in participants and medication practitioners [27]. All studies performed well in the attrition and reporting processes, showing low risk bias in these two domains.

\section{Survival rate of Elderly OSA patients treated with CPAP}

Three studies provided the survival rate of elderly OSA patients treated with CPAP when compared with the counterparts untreated with CPAP. In this pooled analysis, untreated elderly patients exhibited worse survival rate than those with CPAP $(O R=2.22,95 \% \mathrm{Cl}=1.64$ to 3.01 , $\mathrm{P}<0.00001$ ) (Figure 3).

\section{CPAP for CVD risk in elderly OSA patients}

Four studies provided CVD risk data for elderly OSA patients treated with CPAP when compared with the counterparts untreated with CPAP.
In this pooled analysis, treated elderly patients exhibited less cardiovascular risk than those without CPAP ( $\mathrm{RR}=0.49,95 \% \mathrm{Cl}=0.36$ to $0.66, \mathrm{P}<0.00001$ ) (Figure 4 ).

\section{Sleepiness improvement by CPAP for elderly OSA patients}

A total of four studies evaluated the subjective sleepiness by Epworth Sleepiness Scale (ESS) score, and the pooled estimate of effect showed a beneficial effect of CPAP on elderly OSA patients, as assessed by the reduction in $\varepsilon S S$ scores (weighted mean difference 1.70, 95\% Cl: 1.11, 2.30, P<0.00001, Figure 5). However, a significant heterogeneity existed, which could not be explained by differences in age, gender ratio, body mass index, study location, the mean number of hours of CPAP per study, or the nature of the control.

Quality of life and neurocognitive function changes after CPAP treatment

Three studies evaluated the effect of CPAP treatment on the quality of life by Québec Sleep Questionnaire (PSQ). Pooled weighted mean difference indicated a statistically significant improvement on all the domains of DSQ (Supplementary Figure 1). However, a significant 


\begin{tabular}{|c|c|c|c|c|c|c|c|c|c|}
\hline \multicolumn{2}{|c|}{$\begin{array}{l}\text { First Author } \\
\text { (Published Year) }\end{array}$} & $\begin{array}{l}\text { Ou P } \\
(2015)\end{array}$ & $\begin{array}{l}\text { Nishihata } \\
\text { y (2015) }\end{array}$ & $\begin{array}{l}\text { McMilliam } \\
\text { A }(2014)\end{array}$ & $\begin{array}{l}\text { Martinez- } \\
\text { Garcia M } \\
(2012) a\end{array}$ & $\begin{array}{l}\text { Martinez- } \\
\text { Garcia M } \\
(2015) b\end{array}$ & $\begin{array}{l}\text { Dalmases } \\
\text { M (2015) }\end{array}$ & $\begin{array}{l}\text { Crawford- } \\
\text { Achour } \varepsilon \\
(2015)\end{array}$ & $\begin{array}{l}\text { * } \\
\text { Medium } \\
\text { months }\end{array}$ \\
\hline \multicolumn{2}{|l|}{ Location } & China & Japan & UK & Spain & Spain & Spain & France & \\
\hline \multicolumn{2}{|l|}{ Study } & Prospective & Retrospective & Prospective & Prospective & Prospective & Pilot & Prospective & \\
\hline \multicolumn{2}{|c|}{ Recruitment period } & $\begin{array}{l}1998.1 .1 \sim \\
2006.12 .31\end{array}$ & $\begin{array}{c}2004.11 \sim \\
2011.7\end{array}$ & $\begin{array}{l}2010.2 .24 \sim \\
2012.5 .30\end{array}$ & $\begin{array}{l}1998.12 ~ \\
2007.12\end{array}$ & - & $\begin{array}{c}2011.11 \sim \\
2013.7\end{array}$ & $\begin{array}{l}2001.1 \sim \\
2002.12\end{array}$ & \\
\hline \multicolumn{2}{|c|}{ Number (M/F) } & $130(104 / 26)$ & $130(93 / 37)$ & $278(229 / 49)$ & $\begin{array}{c}784 \\
(507 / 277)\end{array}$ & $224(153 / 71)$ & $33(23 / 10)$ & $126(77 / 49)$ & \\
\hline \multicolumn{2}{|c|}{ Age $($ mean $\pm S D)$} & $72.8 \pm 6.2$ & $72.8 \pm 5.0$ & $71.1 \pm 4.7$ & $70.7 \pm 4.4$ & $75.5 \pm 3.9$ & $71.3 \pm 5.51$ & $74.8 \pm 1.1$ & \\
\hline \multicolumn{2}{|l|}{ Status } & $\begin{array}{c}\text { Moderate to } \\
\text { Severe }\end{array}$ & $\begin{array}{c}\text { Moderate to } \\
\text { Severe }\end{array}$ & $\begin{array}{c}\text { Moderate to } \\
\text { Severe }\end{array}$ & $\begin{array}{c}\text { Moderate to } \\
\text { Severe }\end{array}$ & Severe & Severe & Severe & \\
\hline \multicolumn{2}{|c|}{$\begin{array}{l}\text { Followed-up, mean } \pm \\
\text { SD (Range) }\end{array}$} & $\begin{array}{c}5 \pm 2.54 \text { years } \\
(1 \sim 8 \text { years })\end{array}$ & $\begin{array}{c}32.9 \pm 23.8 \\
\text { months }\end{array}$ & $\begin{array}{c}\text { (3 12 } \\
\text { months) }\end{array}$ & $\begin{array}{l}69 \text { months* } \\
(1 \sim 130 \\
\text { months })\end{array}$ & 3 months & 3 months & & \\
\hline \multirow{2}{*}{ Treatment } & CPAP & 36 & 64 & 140 & 503 & 115 & 17 & 33 & \\
\hline & No CPAP & 88 & 66 & 138 & 281 & 109 & 16 & 93 & \\
\hline
\end{tabular}

Table 1. Demographic Information.

heterogeneity existed, especially in the domains of diurnal symptoms, nocturnal symptoms, and emotions.

The data of neuropsychological and neurocognitive tests were extracted from three studies and calculated as the weighted mean difference in each subgroup, which showed that CPAP treatment improved the cognitive functions including TMT A, TMT B, and semantic fluency tests, whereas CPAP had no effect on the digit span and digit symbol, as well as in the neuropsychological tests (Supplementary Figure 2).

\section{Discussion}

Several prior meta-analyses have demonstrated the effects of CPAP in patients with OSA, all of which enrolled middle-aged patients. To date, no meta-analysis was carried out to confirm the influence of CPAP on those elderly patients with OSA. The prevalence of OSA is increased in the elders, especially aging over 70 years. Nevertheless, most of these subjects with OSA refused CPAP treatment, whereas many of them did not appear to have a clear understanding of the health consequences of OSA. Hence, several randomized clinical trials were registered hoping to provide clinical evidences to confirm the efficacy of CPAP in elderly OSA patients. In this situation, we carried out this meta-analysis to validate whether CPAP treatment influences clinical outcomes and quality of life in elderly patients with OSA. This is the first metaanalysis in a specific aged population to our best knowledge.

The main findings of this meta-analysis were that, in elderly patients with moderate to severe sleep apnea, CPAP treatment increased the survival rate. Both Ou et al. [25] and Nishihata et al. [26] compared

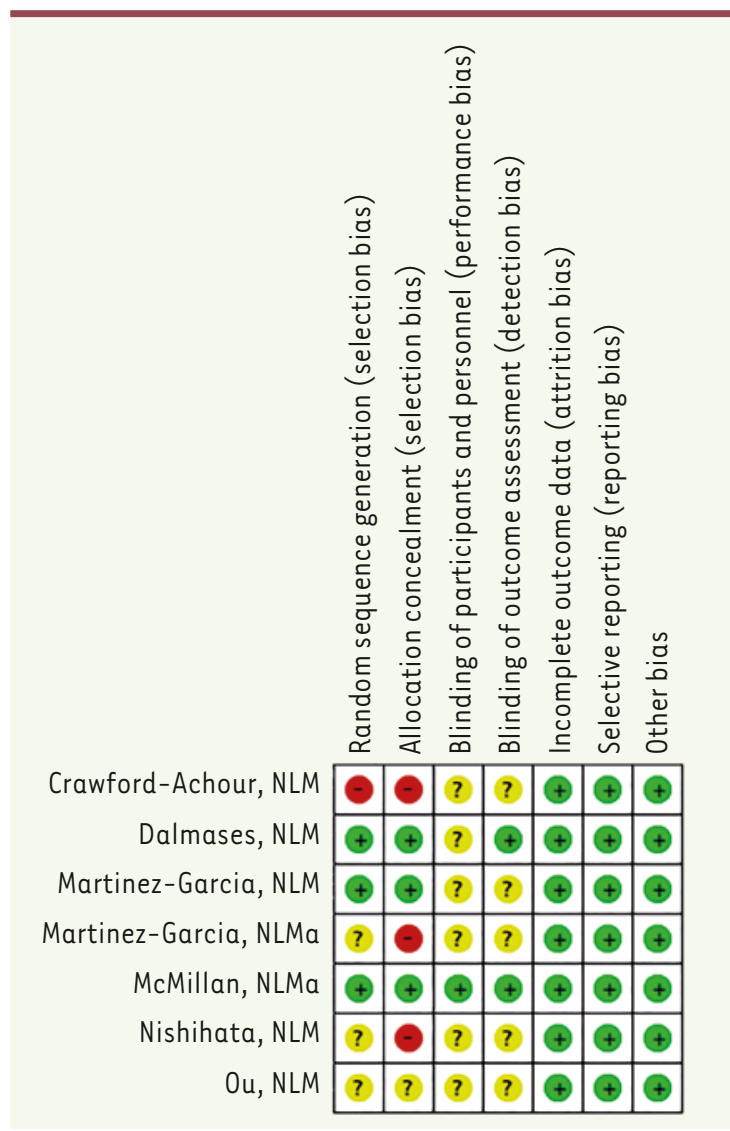

Figure 2. Risk of bias summary. 


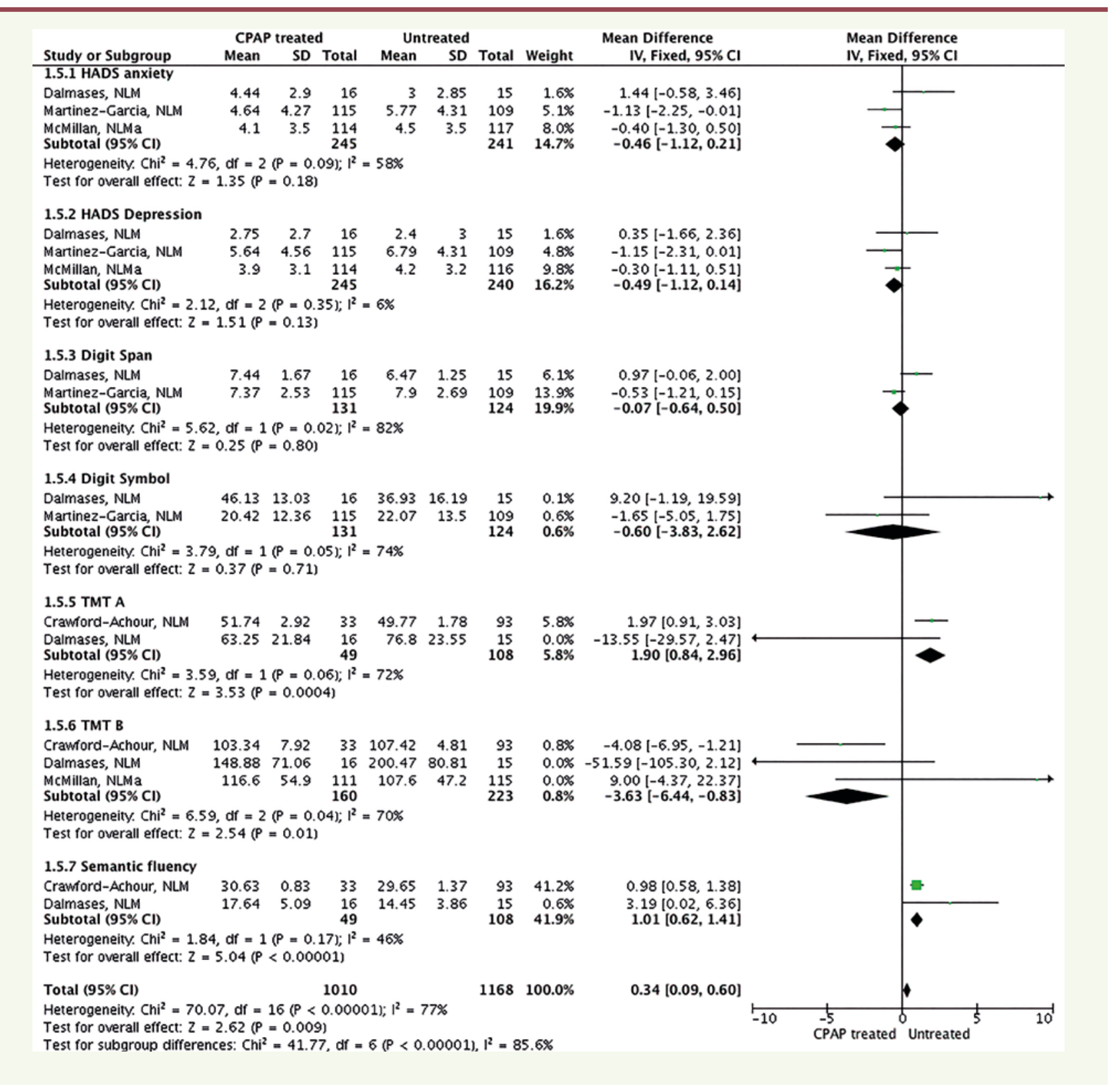

Supplementary Figure 2. Forest plot for neurocognitive test.

the survival rate between the CPAP treatment and untreated groups. Both of these two randomized clinical trials found that the event-free survival rate was significantly decreased in the CPAP treatment group, and the adjusted Kaplan-Meier survival curves exhibited significantly decreased mortality in the CPAP treatment group. Further comparison showed that CVD events were statistically decreased after CPAP treatment. However, only small changes in the total cholesterol and no improvement in blood pressure were detected in the McMillan and colleagues study [31]. Although CVD events are the first predictors of death for these elderly OSA people, $\mathrm{Ou}$ and his colleagues also pointed out that diabetes might be an independent risk factor for them [25]. Considering that aging is profoundly associated with multiple physical or organ deteriorations, comorbidities are inevitable factors to predict all-cause mortality. Marrone et al. reported that CPAP users had a better survival than non-users among subjects with comorbidities taken alone instead of those without comorbidities [32].

Previous studies described that OSA would result in the cognitive deficits and neuropsychological impairments of executive functions, attention, and working memory among old-aged patients [33, 34]. Prior investigation examined the short-term effect of CPAP treatment on neurocognitive performance in elderly subjects with OSA; the results indicated a modest improvement in 


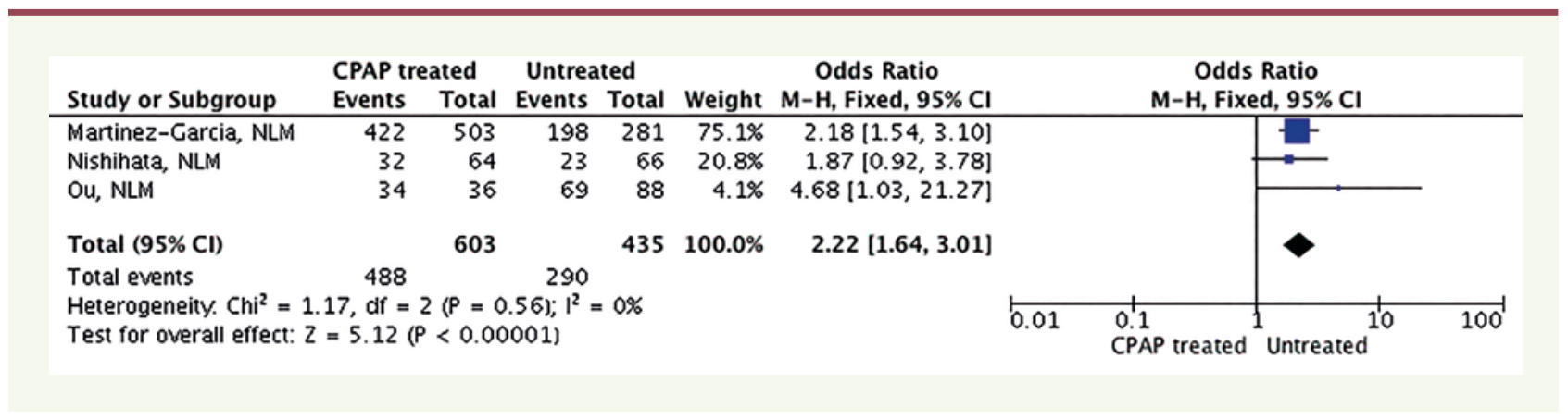

Figure 3. Forest plot of comparison for Survival rate.

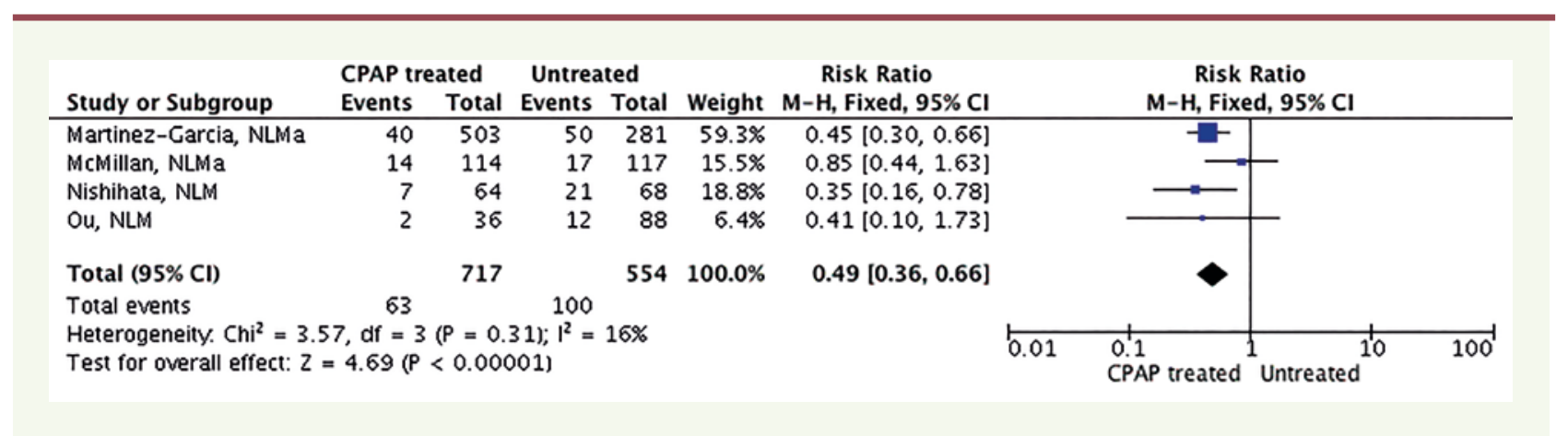

Figure 4. Forest plot of comparison for CVD rate.

executive functioning [35]. Afterwards, several well-designed RCTs were conducted in confirming the impact of CPAP on cognitive difficulties and neuropsychological problems among those elderly OSA patients $[27,29,30]$. Pooled weighted mean difference showed that CPAP treatment has partial effect on the cognitive functions evidenced in TMT A, TMT B, and semantic fluency tests, whereas it has no impact on the neuropsychological symptoms, such as depression and anxiety. Dalmases et al. recorded resting-state connectivity alterations associated with CPAP treatment in elderly patients [29]. They showed a significant increase in the intensity of connectivity in the right middle frond gyrus ( $r M F G$ ), but no association was seen between cognition and increased connectivity within rMFG. The other two studies reinforce that specific regions, such as the hippocampus or frontal structures affecting memory, attention, and executive functioning, were neuroimaging-scanned showing structure alterations associated with cognitive function improvements in CPAP treated subjects [36, 37]. Nevertheless, their work shows new insight into the effect of CPAP on brain function and structure changes among elderly patients with OSA, as well as in the context of aging.

From the standpoint of quality of life, two kinds of measurements, QSQ and the European Quality of Life-5 Dimensions ( $(Q-5 D)$, were applied in the included RCTs. CPAP treatment improves the quality of life measured by $\mathrm{QS} Q$, including day-time and night-time symptoms and social and emotional domains. However, only one study used $\varepsilon Q-5 \rho$ in the McMillan study; CPAP was shown to improve quality of life both generic and disease specific [31]. To date, only one RCT assessed whether CPAP treatment improved pain tolerance in elderly patients with OSA. It was found that CPAP treatment had an analgesic effect and increased the pain tolerance threshold in the elderly [38]. Currently, pain processing can be interfered by poor sleep and restored sleep. Referred in this meta-analysis, CPAP treatment alleviated sleepiness problems in the value of ESS scores. In this context, our results indicate that CPAP treatment could have more benefits in the elderly patients with OSA suffering from chronic pain. Some limitations of this meta-analysis need to be discussed. First, the follow-up duration time was inconsistent between included trials. More importantly, the duration time has been supposed to have opposite impact on the clinical outcomes, especially the survival time and neuropsychological performance. Long-term follow-up is encouraged to evaluate the survival rate, increasing the prognosis accuracy, whereas it is a major concern in terms of neuropsychological assessment. The reason is because, during this long follow-up period, neuro- 


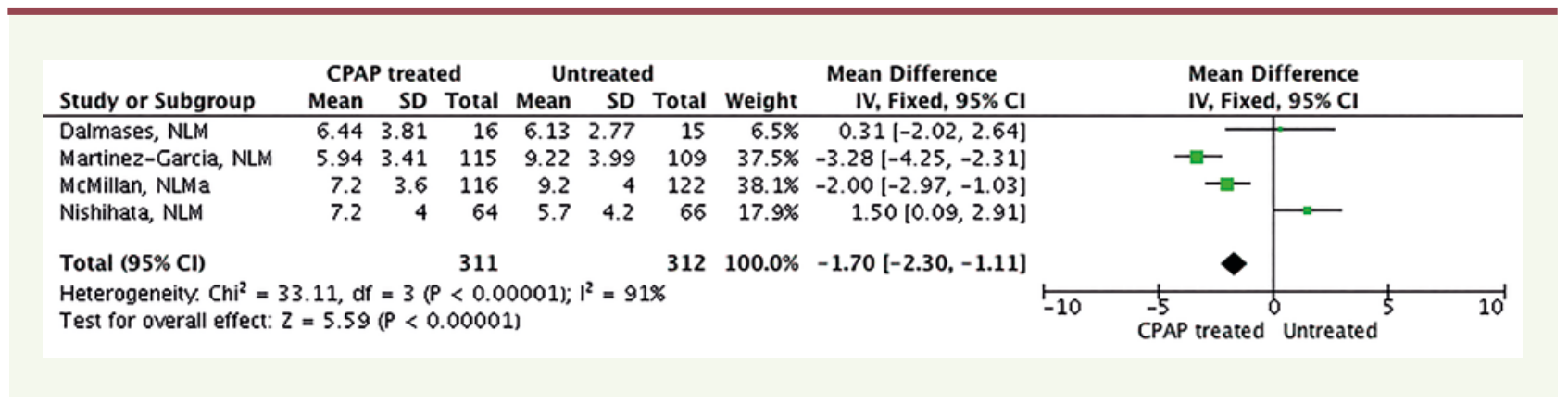

Figure 5. Forest plot for ESS score.

logical modifications cannot be easily ignored and not because of another pathophysiological process considering the physical specialty of the elderly population. Also, there was a concern about i) a possible blinding bias, ii) that CPAP was a physical device and that no sham CPAP was used in the control group among enrolled RCTs, which needs a strict design and performance to exclude the placebo bias. Finally, cognitive and neuropsychological tests were inconsistently performed in the enrolled RCTs.

In conclusion, OSA should be widely accepted as an independent risk factor for the elderly population, and OSA has profound healthy effects leading to multiple comorbidities. CPAP treatment for the elderly patients with OSA achieves improvements in the event-free survival rate and the occurrence of CVD events. Further large-scale, well-designed RCTs are needed to confirm the CPAP influence in the context of comorbidities associated with aging problem, as well as the cognitive performance and neuropsychological impairments. $\diamond$

\section{FUNDING}

Pu-dong Health Bureau of Shanghai provided financial support in the form of key discipline construction project. The sponsor had no role in the design or conduct of this research.

\section{CONFLICT OF INTEREST}

All authors claimed no conflict of interest.

\section{ETHICAL APPROVAL}

All procedures performed in studies involving human participants were in accordance with the ethical standards of the research committee of Shanghai Seventh People's hospital and with the 1964 Helsinki declaration and its later amendments.

\section{INFORMED CONSENT}

Informed consent was obtained from all individual participants included in this study.

\section{REFERENCES}

1. Young $\mathrm{T}$, et al. Predictors of sleep-disordered breathing in community-dwelling adults: the Sleep Heart Health Study. Arch Intern Med 2002; 162(8): 893-900.

2. Young $\mathrm{T}$, et al. The occurrence of sleep-disordered breathing among middle-aged adults. $N$ EnglJ Med 1993; 328(17): 1230-5.

3. Lavie P, Lavie L. Unexpected survival advantage in elderly people with moderate sleep apnoea.J Sleep Res 2009; 18(4): 397-403.

4. Jordan AS, McSharry DG, Malhotra A. Adult obstructive sleep apnoea. Lancet 2014; 383(9918): 736-47.

5. Marin JM, et al. Long-term cardiovascular outcomes in men with obstructive sleep apnoeahypopnoea with or without treatment with continuous positive airway pressure: an observational study. Lancet 2005; 365(9464): 1046-53.
6. Peppard PE, et al. Prospective study of the association between sleepdisordered breathing and hypertension. N Engl J Med 2000; 342(19): $1378-$ 84.

7. Barbe et al. Automobile accidents in patients with sleep apnea syndrome. An epidemiological and mechanistic study. Am J Respir Crit Care Med 1998; 158(1): 18-22.

8. Teran-Santos J, Jimenez-Gomez A, Cordero-Guevara J. The association between sleep apnea and the risk of traffic accidents. Cooperative Group Burgos-Santander. N EnglJ Med 1999; 340(11): 847-51.

9. Malhotra A, Loscalzo J. Sleep and cardiovascular disease: an overview. Prog Cardiovasc Dis 2009; 51(4): 279-84.

10. Gozal D, Kheirandish-Gozal L. Cardiovascular morbidity in obstructive sleep apnea: oxidative stress, inflammation, and much more. Am J Respir Crit Care Med 2008; 177(4): 369-75.

11. Lavie L. Oxidative stress: a unifying paradigm in obstructive sleep apnea and comorbidities. Prog Cardiovasc Dis 2009. 51(4): 303-12.

12. Nieto FJ, et al. Association of sleep-disordered breathing, sleep apnea, and hypertension in a large community-based study. Sleep Heart Health Study. JAMA 2000; 283(14): 1829-36.

13. Arias MA, et al. Obstructive sleep apnea syndrome affects left ventricular diastolic function: effects of nasal continuous positive airway pressure in men. Circulation 2005; 112(3): 375-83.

14. Laaban JP, et al. Left ventricular systolic dysfunction in patients with obstructive sleep apnea syndrome. Chest 2002;. 122(4): 1133-8.

15. Yaggi HK, et al. Obstructive sleep apnea as a risk factor for stroke and death. $N$ Engl J Med 2005; 353(19): 2034-41.

16. Sforza $\varepsilon$, Roche F. Sleep apnea syndrome and cognition. Front Neurol 2012; 3: 87.

17. Ancoli-Israel S. Sleep apnea in older adults: is it real and should age be the determining factor in the treatment decision matrix? Sleep Med Rev 2007; 11(2): 83-5.

18. Takama N, Kurabayashi M. Influence of untreated sleep-disordered breathing on the long-term prognosis of patients with cardiovascular disease. Am J Cardiol 2009; 103(5): 730-4.

19. Martinez-Garcia MA, et al. Cardiovascular mortality in obstructive sleep apnea in the elderly: role of long-term continuous positive airway pressure treatment: a prospective observational study. Am J Respir Crit Care Med 2012; 186(9): 909-16.

20. Sanders MH, et al. Positive pressure therapy: a perspective on evidencebased outcomes and methods of application. Proc Am Thorac Soc 2008; 5(2): 161-72.

21. Duran-Cantolla J, et al. Continuous positive airway pressure as treatment for systemic hypertension in people with obstructive sleep apnoea: randomised controlled trial. BMJ 2010; 341: c5991.

22. Martinez-Garcia MA, et al. Continuous positive airway pressure treatment reduces mortality in patients with ischemic stroke and obstructive sleep apnea: a 5-year follow-up study. Am J Respir Crit Care Med 2009; 180(1): 36-41.

23. Barbe F, et al. Long-term effect of continuous positive airway pressure in hypertensive patients with sleep apnea. Am J Respir Crit Care Med 2010; 181(7): 718-26.

24. Campos-Rodriguez F, et al. Mortality in obstructive sleep apnea-hypopnea patients treated with positive airway pressure. Chest 2005; 128(2): 624-33.

25. Ou $Q$, et al. Continuous Positive Airway Pressure Treatment Reduces Mortality in Elderly Patients with Moderate to Severe Obstructive Severe Sleep Apnea: A Cohort Study. PLoS One 2015; 10(6): e0127775. 


\section{REFERENCES}

26. Nishihata $Y$, et al. Continuous positive airway pressure treatment improves cardiovascular outcomes in elderly patients with cardiovascular disease and obstructive sleep apnea. Heart Vessels 2015; 30(1): 61-9.

27. McMillan A, et al. Continuous positive airway pressure in older people with obstructive sleep apnoea syndrome (PREDICT): a 12-month, multicentre, randomised trial. Lancet Respir Med 2014 2(10): 804-12.

28. Martinez-Garcia MA, et al. Obstructive sleep apnoea in the elderly: role of continuous positive airway pressure treatment. Eur Respir J 2015; 46(1): 142-51.

29. Dalmases $M$, et al. Effect of CPAP on Cognition, Brain Function, and Structure Among Elderly Patients With OSA: A Randomized Pilot Study. Chest 2015; 148(5): 1214-23.

30. Crawford-Achour $\varepsilon$, et al. Protective Effect of Long-Term CPAP Therapy on Cognitive Performance in Elderly Patients with Severe OSA: The PROOF Study. J Clin Sleep Med 2015; 11(5): 519-24.

31. McMillan A, et al. A multicentre randomised controlled trial and economic evaluation of continuous positive airway pressure for the treatment of obstructive sleep apnoea syndrome in older people: PREDICT. Health Technol Assess 2015; 19(40): 1-188.
32. Marrone 0 , et al. Comorbidities and survival in obstructive sleep apnoea beyond the age of 50. Eur J Clin Invest 2013; 43(1): 27-33.

33. Yaffe $K$, et al. Sleep-disordered breathing, hypoxia, and risk of mild cognitive impairment and dementia in older women. JAMA 2011; 306(6): 613-9.

34. Ju G, et al. Effects of sleep apnea syndrome on delayed memory and executive function in elderly adults. J Am Geriatr Soc 2012; 60(6): 1099-103.

35. Aloia MS, et al. Neuropsychological changes and treatment compliance in older adults with sleep apnea. J Psychosom Res 2003; 54(1): 71-6.

36. Canessa $\mathrm{N}$, et al. Obstructive sleep apnea: brain structural changes and neurocognitive function before and after treatment. Am J Respir Crit Care Med 2011; 183(10): 1419-26.

37. Fotuhi M, Do D, Jack C. Modifiable factors that alter the size of the hippocampus with ageing. Nat Rev Neurol 2012; 8(4): 189-202.

38. Onen SH, et al. Pain tolerance and obstructive sleep apnea in the elderly. J Am Med Dir Assoc 2010; 11(9): 612-6. 